\title{
Almost periodic solutions of partial differential equations with delay
}

\author{
Hernán R Henríquez ${ }^{1}$, Claudio Cuevas $^{2 *}$ and Alejandro Caicedo ${ }^{3}$
}

\section{"Correspondence:}

cch@dmat.ufpe.br

2Departmento of Matemática,

Universidade Federal of

Pernambuco, Av. Prof. Luiz Freire,

S/N, Recife, PE 50540-740, Brazil

Full list of author information is

available at the end of the article

\begin{abstract}
In this paper we study the existence of almost periodic solutions for linear retarded functional differential equations with finite delay and values in a Banach space. We relate the existence of almost periodic solutions with the stabilization of distributed control systems. We apply our results to transport models and to the wave equation. MSC: Primary 34K14; 34K30; secondary 35R10; 47D06; 93B05

Keywords: retarded functional differential equations in abstract spaces; almost periodic functions; semigroups of operators; compact operators; controllability of systems
\end{abstract}

\section{Introduction}

Motivated by the fact that abstract retarded functional differential equations (abbreviated, ARFDE) arise in many areas of applied mathematics, this type of equations has received much attention in recent years [1-4]. Some applications and numerical methods for equations where the operator acting on the delay term is bounded have been studied in [5-8].

ARFDEs where the operator acting on delay term is unbounded have been studied in some works. For equations on Hilbert spaces, we refer to [9] and the references therein. Moreover, ARFDEs on general Banach spaces where the operator acting on the delay term is unbounded have been studied in a few works. For information of the reader, we refer to [10-13]. These studies are aimed at regular solutions of partial differential equations. Moreover, the study of more general solutions of ARFDEs where the operator acting on delay term is unbounded has been addressed in recent work. Stability aspects and differences schemes for approximate solutions have been treated in [14-20].

In particular, the problem of existence of almost periodic and asymptotically almost periodic solutions has been considered by several authors. We refer the reader to the book [21] and to the papers [22-30] and the references listed therein for recent information on this subject. Our objective is to establish existence of almost periodic mild solutions for a class of linear ARFDE of first order.

Throughout this work, we denote by $X$ a complex Banach space endowed with a norm $\|\cdot\|$. Henceforth, we represent by $A$ the infinitesimal generator of a strongly continuous semigroup of bounded linear operators $T(t)$ on $X$, and $C$ stands for the space of continuous functions $C([-r, 0] ; X), r>0$, provided with the norm of uniform convergence. We will be

(c) 2015 Henríquez et al.; licensee Springer. This is an Open Access article distributed under the terms of the Creative Commons Attribution License (http://creativecommons.org/licenses/by/4.0), which permits unrestricted use, distribution, and reproduction in any medium, provided the original work is properly credited. 
concerned with the existence of almost periodic solutions to the Cauchy problem

$$
x^{\prime}(t)=A x(t)+L\left(x_{t}\right)+f(t), \quad t \in \mathbb{R},
$$

where $x(t) \in X$, the function $x_{t}:[-r, 0] \rightarrow X$, which denotes the segment of $x(\cdot)$ at $t$, is given by $x_{t}(\theta)=x(t+\theta), L: C \rightarrow X$ is a bounded linear map, and $f: \mathbb{R} \rightarrow X$ is an appropriate function. The usual condition for studying the existence of almost periodic solutions for this problem is that the semigroup $T(\cdot)$ is compact. For example, the problem of existence of almost automorphic solutions has been studied recently by Ezzinbi and N'Guérékata [25] using this condition. Our aim in this paper is to establish the existence of almost periodic solutions for a class of equations in which the semigroup $T(\cdot)$ is not compact.

As a model we consider the wave equation with delay

$$
\begin{aligned}
& \frac{\partial^{2}}{\partial t^{2}} w(\xi, t)+\beta \frac{\partial}{\partial t} w(\xi, t)=\frac{\partial^{2}}{\partial \xi^{2}} w(\xi, t)+\int_{-r}^{0} d_{\theta} N(\theta) w(\xi, t+\theta)+b(\xi, t), \\
& w(0, t)=w(\pi, t)=0
\end{aligned}
$$

for $0 \leq \xi \leq \pi$ and $t \geq 0$, where the scalar function $N(\cdot)$ has bounded variation on [-r, 0] and $b(\cdot)$ is an appropriate function. Usually this type of linear equations arise from linearization of semilinear ones [4].

For the necessary concepts related with the abstract Cauchy problem and the theory of strongly continuous semigroup of operators we refer to Engel and Nagel [31] and Pazy [32]. We only mention here a few concepts and results directly related with our development. Let $G(t)$ be a strongly continuous semigroup defined on a Banach space $X$ with infinitesimal generator $D$. We say that $G$ is strongly stable if $G(t) x \rightarrow 0, t \rightarrow \infty$, for all $x \in X$ and we say that $G$ is uniformly stable if $\|G(t)\| \rightarrow 0, t \rightarrow \infty$. Moreover, we employ the terminology and notations for spectral bound $s(D)$, growth bound $\omega_{0}(G)$ and essential growth bound $\omega_{\text {ess }}(G)$ from [31]. Specifically, $s(D)=\sup \{\operatorname{Re}(\lambda): \lambda \in \sigma(D)\} ; \omega_{0}(G)=\lim _{t \rightarrow \infty} \frac{\ln \|G(t)\|}{t}$ and $\omega_{\text {ess }}(G)=\lim _{t \rightarrow \infty} \frac{\ln \|G(t)\|_{\text {less }}}{t}$, where the symbol $\|\cdot\|_{\text {ess }}$ denotes the essential norm of an operator. Consequently, in terms of these notations, $G$ is uniformly stable if, and only if, $\omega_{0}(G)<0$.

For completeness we also mention here that a strongly continuous semigroup $G(t)$ is said to be compact if $G(t)$ is a compact operator for all $t>0$ and that $G$ is said to be quasicompact if there is $t_{0}>0$ and a compact operator $R$ such that $\left\|G\left(t_{0}\right)-R\right\|<1$. We collect in the following lemma a pair of fundamental results [31, Corollary IV.2.11, Proposition V.3.5] for our further development.

Lemma 1.1 The following conditions are fulfilled.

(i) The semigroup $G$ is quasi-compact if and only if $\omega_{\mathrm{ess}}(G)<0$.

(ii) $\omega_{0}(G)=\max \left\{\omega_{\text {ess }}(G), s(D)\right\}$.

Throughout this work we denote by $\mathcal{L}(X)$ the Banach algebra of bounded linear operators defined on $X$ and by $X^{*}$ the dual space of $X$. For a linear operator $A$ with domain $D(A)$ and range $\mathcal{R}(A)$ in $X$, we represent by $\sigma(A)$ (resp. $\sigma_{p}(A), \rho(A)$ ) the spectrum (respectively point spectrum, resolvent set) of $A$. For $\lambda \in \rho(A)$ we set $R(\lambda, A)=(\lambda I-A)^{-1}$ for the resolvent operator of $A$. Finally, if $D(A)$ is dense in $X$, then $A^{\prime}$ denotes the dual operator of $A$ [33]. 
This paper is organized as follows. In Section 2 we have collected some technical results about spectral properties of ARFDE, most of which are included in [4, 31, 34], in Section 3 we apply these properties to study the existence of almost periodic solutions of ARFDE, and in Section 4 we have included some applications of our results.

\section{Preliminaries}

Throughout the rest of this paper $A: D(A) \rightarrow X$ is the infinitesimal generator of a semigroup of bounded linear operators $T(t)$ on $X$ and $L: C \rightarrow X$ is a bounded linear map defined by

$$
L(\varphi)=\int_{-r}^{0} d_{\theta} N(\theta) \varphi(\theta)
$$

where $N:[-r, 0] \rightarrow \mathcal{L}(X)$ is a function of bounded variation which is left continuous on $(-r, 0)$ and $N(0)=N(-r)=0$, and $f: \mathbb{R} \rightarrow X$ is a continuous function. We refer to Engel and Nagel [31], Travis and Webb [34] and Wu [4] for the basic properties of the ARFDE

$$
x^{\prime}(t)=A x(t)+L\left(x_{t}\right)+f(t), \quad t \geq \sigma .
$$

We only mention here that (2.2) with the initial condition

$$
x_{\sigma}=\varphi \in C
$$

has a unique mild solution $x=x(\cdot, \sigma, \varphi, f)$. This means that $x:[\sigma-r, \infty) \rightarrow X$ is a continuous function that verifies $(2.3)$ and the restriction of $x(\cdot)$ on $[\sigma, \infty)$ satisfies the integral equation

$$
x(t)=T(t-\sigma) \varphi(0)+\int_{\sigma}^{t} T(t-s)\left(L\left(x_{s}\right)+f(s)\right) d s, \quad t \geq \sigma .
$$

In particular, if $x(\cdot, \varphi)$ denotes the mild solution of the homogeneous problem

$$
\begin{aligned}
& x^{\prime}(t)=A x(t)+L\left(x_{t}\right), \quad t \geq 0, \\
& x_{0}=\varphi \in C,
\end{aligned}
$$

the solution operator $V(t)$ defined by $V(t) \varphi=x_{t}(\cdot, \varphi)$ is a strongly continuous semigroup of bounded linear operators on $C$ (see [4]). We will represent by $A_{V}$ its infinitesimal generator. Moreover, it is well known that $V$ satisfies the following translation property.

Lemma 2.1 Under the preceding conditions,

$$
[V(t) \varphi](\theta)= \begin{cases}{[V(t+\theta) \varphi](0),} & t+\theta \geq 0, \\ \varphi(t+\theta), & t+\theta \leq 0 .\end{cases}
$$

We denote by $W(t)$ the solution operator corresponding to $L=0$. It is clear that $W(t)$ is given by

$$
[W(t) \varphi](\theta)= \begin{cases}T(t+\theta) \varphi(0), & -t \leq \theta \leq 0, \\ \varphi(t+\theta), & -r \leq \theta<-t .\end{cases}
$$


The system (2.5) is said to be (asymptotically) stable if the semigroup $V(t)$ is uniformly stable. Consequently, it follows from Lemma 1.1 that the study of the asymptotic stability of the system (2.5) is reduced to the study of spectral properties of the solution semigroup $V(t)$.

We are in a position to establish the first result about asymptotic behavior of the solution semigroup.

Theorem 2.1 Assume that the semigroup $T(t)$ is uniformly stable and that the operator $T(t) L: C \rightarrow X$ is compact for all $t>0$. Then the semigroup $V(t)$ is quasi-compact.

Proof We define the operator $U(t): C \rightarrow C$ for $t \geq 0$ by

$$
[U(t) \varphi](\theta)= \begin{cases}\int_{0}^{t+\theta} T(t+\theta-s) L(V(s) \varphi) d s, & -t \leq \theta \leq 0, \\ 0, & -r \leq \theta<-t .\end{cases}
$$

It is clear from (2.4) that

$$
V(t)=W(t)+U(t), \quad t \geq 0,
$$

which implies that $U(t)$ is a bounded linear operator. Moreover, from [35, Theorem 1] we can assert that $U(t)$ is a compact operator. On the other hand, there exist constants $M \geq 1$ and $\alpha>0$ such that $\|T(t)\| \leq M e^{-\alpha t}$ for all $t \geq 0$. Hence, for $t \geq r$ we have

$$
\begin{aligned}
\|W(t) \varphi\| & =\sup _{-r \leq \theta \leq 0}\|T(t+\theta) \varphi(0)\| \leq M \sup _{-r \leq \theta \leq 0} e^{-\alpha(t+\theta)}\|\varphi(0)\| \\
& \leq M e^{\alpha r} e^{-\alpha t}\|\varphi\|,
\end{aligned}
$$

which implies that $W(t)$ is uniformly stable.

There are many interesting situations in which the semigroup $T(t)$ is not compact but the operator $T(t) L: C \rightarrow X$ is compact for $t>0$. Next we mention a pair of general cases:

(i) The operator $L: C \rightarrow X$ is compact. For instance, $L(\varphi)=\sum_{i=1}^{k} A_{i} \varphi\left(-r_{i}\right)$, where $A_{i}$ : $X \rightarrow X, i=1, \ldots, k$, are compact linear operators, or $L(\varphi)=\int_{-r}^{0} N(\theta) \varphi(\theta) d \theta$, where $N$ : $[-r, 0] \rightarrow \mathcal{L}(C)$ is a map continuous for the norm of operators and $N(\theta)$ is a compact operator for each $-r \leq \theta \leq 0$. As a matter of fact, this property is verified under more general conditions in $N$. In Section 4 we present some concrete examples.

(ii) A more general situation is the following. Assume that there exists a topological decomposition of $X=X_{0} \oplus X_{1}$, where $X_{i}$ are invariant spaces under $T(t)$, and $X_{1}$ has finite dimension. Let $P_{0}$ be the projection on $X_{0}$ with kernel $X_{1}$. If $T(t) P_{0} L$ is compact, then the product $T(t) L$ is also compact.

Combining Theorem 2.1 with Theorem V.3.7 in [31] we can establish the following property of asymptotic behavior for the solution semigroup associated to the homogeneous problem (2.5)-(2.6).

Corollary 2.1 Assume that the semigroup $T(t)$ is uniformly stable and that the operator $T(t) L: C \rightarrow X$ is compact for all $t>0$. Then the semigroup $V(t)$ is uniformly stable if and only if $\sup \operatorname{Re} \sigma_{p}\left(A_{V}\right)<0$. 
To study the $\sigma_{p}\left(A_{V}\right)$, the following spectral property is well known (see for example [26] and the references cited therein). We include it here for completeness. For $\lambda \in \mathbb{C}$, let $L_{\lambda}: X \rightarrow X$ and $\Delta(\lambda): D(A) \rightarrow X$ be the linear operators defined by

$$
\begin{aligned}
& L_{\lambda} x=L\left(e^{\lambda \theta} x\right), \quad x \in X, \\
& \Delta(\lambda) x=\lambda x-A x-L_{\lambda} x, \quad x \in D(A) .
\end{aligned}
$$

Lemma 2.2 Let $\lambda \in \mathbb{C}$. Then $\lambda \in \sigma_{p}\left(A_{V}\right)$ if and only if there is $u \in D(A), u \neq 0$, such that $\Delta(\lambda) u=0$. In this case the function $\varphi=e^{\lambda \theta} u$ is the eigenvector of $A_{V}$ corresponding to $\lambda$. Moreover, if $\lambda \in \sigma\left(A+L_{\lambda}\right)$, then $\lambda \in \sigma\left(A_{V}\right)$.

We return to the nonhomogeneous equation (2.2). It is clear that this equation can be considered as an equation with infinite delay. In fact, we can extend the map $N$ to $(-\infty, 0]$ by defining $N(\theta)=0$ for $\theta<-r$. Besides, we can consider a uniform fading memory space $\mathcal{B}$ such that $\mathcal{B} \subseteq C((-\infty, 0], X)$ and $L(\varphi)=\int_{-\infty}^{0} d_{\theta} N(\theta) \varphi(\theta)$, for $\varphi \in \mathcal{B}$, defines a bounded linear map from $\mathcal{B}$ into $X$. This property allows us to apply the theory developed in [36] to represent the mild solution of (2.2). It has been proved by Hino et al. [36] that the solution can be expressed by means of the variation of constants formula. Next we adopt the notations used in this paper. Let $\Gamma^{n}: X \rightarrow \mathcal{B}$ be defined by

$$
\Gamma^{n}(\theta) x= \begin{cases}(n \theta+1) x, & -1 / n \leq \theta \leq 0, \\ 0, & -\infty<\theta<-1 / n .\end{cases}
$$

If $f$ is a continuous function, the formula [36, Theorem 3.1]

$$
x_{t}(\cdot, \sigma, \varphi, f)=V(t-\sigma) \varphi+\lim _{n \rightarrow \infty} \int_{\sigma}^{t} V(t-s) \Gamma^{n} f(s) d s
$$

gives the mild solution of (2.2)-(2.3) in the phase space. Moreover, applying [36, Proposition 4.2 ] we can establish the following property.

Remark 2.1 Assume that semigroup $V(t)$ is quasi-compact. In this case the set $\Lambda=\{\lambda \in$ $\left.\sigma\left(A_{V}\right): \operatorname{Re}(\lambda) \geq 0\right\}$ is finite and consists of poles of $R\left(\cdot, A_{V}\right)$ with finite algebraic multiplicity [31, Theorem V.3.7]. Therefore, the space $C$ is decomposed as

$$
C=P_{\Lambda} \oplus Q_{\Lambda},
$$

where $P_{\Lambda}$ and $Q_{\Lambda}$ are spaces invariant under $V(t)$ and the space $P_{\Lambda}$ is the range of the spectral projection $\Pi^{P}$ corresponding to $\Lambda$. Consequently, $P_{\Lambda}$ consists of the generalized eigenvectors corresponding to the eigenvalues $\lambda_{i} \in \Lambda$. Specifically, if $\Lambda=\left\{\lambda_{1}, \lambda_{2}, \ldots, \lambda_{m}\right\}$, then

$$
P_{\Lambda}=\bigoplus_{i=1}^{m} \operatorname{ker}\left(\lambda_{i} I-A_{V}\right)^{k_{i}}
$$

for certain $k_{i} \in \mathbb{N}$. We denote by $V^{P}(t)$ (respectively, $V^{Q}(t)$ ) the restriction of $V(t)$ on $P_{\Lambda}$ (respectively, on $Q_{\Lambda}$ ). Similarly, $A_{V}^{P}$ and $A_{V}^{Q}$ represent the restrictions of $A_{V}$ on $P_{\Lambda}$ and $Q_{\Lambda}$, 
respectively. Since $P_{\Lambda}$ is a space of finite dimension $d$, the semigroup $V^{P}(t)$ is uniformly continuous and $A_{V}^{P}$ is a bounded linear operator defined on $P_{\Lambda}$. Let $\varphi^{1}, \varphi^{2}, \ldots, \varphi^{d}$ be a basis of $P_{\Lambda}$. We set $\Phi=\left(\varphi^{1}, \varphi^{2}, \ldots, \varphi^{d}\right)$. Consequently, proceeding as usual in the theory of functional differential equations [37], there is a $d \times d$ matrix $G$ such that $\Phi(\theta)=\Phi(0) e^{G \theta}$, for $-r \leq \theta \leq 0, V^{P}(t) \Phi=\Phi e^{G t}$, for $t \geq 0$, and $\sigma_{p}(G)=\Lambda$. Let $\Psi$ be the dual basis of $\Phi$ associated to the decomposition (2.10), as constructed in [36]. In these conditions, it has been established in [36, Proposition 4.2] that there exists $x^{*}=\operatorname{col}\left(x_{1}^{*}, x_{2}^{*}, \ldots, x_{d}^{*}\right) \in X^{* d}$ such that the projection $x_{t}^{P}=\Pi^{P} x_{t}$ on $P_{\Lambda}$ of the solution of (2.2) is given by $x_{t}^{P}=\Phi z(t)$, where the $d$-vector $z(t)$ satisfies the ordinary differential equation

$$
z^{\prime}(t)=G z(t)+\left\langle x^{*}, f(t)\right\rangle, \quad t \geq \sigma .
$$

On the other hand, in this work we employ the concept of almost periodic function in the sense of Bohr. This means that a function $f: \mathbb{R} \rightarrow X$ is almost periodic if it is continuous and for every $\varepsilon>0$ there is a relatively dense set $P_{\varepsilon}$ in $\mathbb{R}$ such that

$$
\|f(t+\tau)-f(t)\| \leq \varepsilon
$$

for all $t \in \mathbb{R}$ and $\tau \in P_{\varepsilon}$. For the properties of almost periodic functions we refer the reader to $[38,39]$. We denote by $A P(X)$ the space consisting of the functions $x: \mathbb{R} \rightarrow X$ which are almost periodic.

To complete this section, we establishes formally the following concept.

Definition 2.1 A continuous function $x: \mathbb{R} \rightarrow X$ is said to be a mild solution of (1.1) if for each $\sigma \in \mathbb{R}$ the restriction $x:[\sigma, \infty) \rightarrow X$ is a mild solution of (2.2) with initial condition $x_{\sigma}$ at $t=\sigma$.

\section{Existence of almost periodic solutions}

In this section we turn our attention to the existence of almost periodic solutions of (1.1). Throughout this section we assume that $A$ and $L$ satisfy the general conditions considered in Section 2 and that $f: \mathbb{R} \rightarrow X$ is a continuous function.

If the semigroup $V$ is quasi-compact, we can apply the properties and notations introduced in Remark 2.1 in relation with the homogeneous equation (2.5). In particular, we set $\Lambda=\left\{\lambda \in \sigma_{p}\left(A_{V}\right): \operatorname{Re}(\lambda) \geq 0\right\}$. A direct application of [36, Theorem 4.3] gives the following property.

Proposition 3.1 Assume that the semigroup $T$ is uniformly stable and that the operator $T(t) L$ is compact for $t>0$. If $x: \mathbb{R} \rightarrow X$ is a mild solution of $(1.1)$ on $\mathbb{R}$, then $z(t)=\left\langle\Psi, x_{t}\right\rangle$ is a solution of the differential equation

$$
z^{\prime}(t)=G z(t)+\left\langle x^{*}, f(t)\right\rangle, \quad t \in \mathbb{R}
$$

Conversely, iff is a bounded function on $\mathbb{R}$ and $z(\cdot)$ is a solution of (3.1), then the function $x: \mathbb{R} \rightarrow$ X given by

$$
x(t)=\left[\Phi z(t)+\lim _{n \rightarrow \infty} \int_{-\infty}^{t} V^{Q}(t-s) \Pi^{Q}\left(\Gamma^{n} f(s)\right) d s\right](0), \quad t \in \mathbb{R},
$$

is a mild solution of (1.1). 
Now we are in a position to establish the main result of this work.

Theorem 3.1 Assume that the semigroup $T$ is uniformly stable and that the operator $T(t) L$ is compact for $t>0$. Let $f: \mathbb{R} \rightarrow X$ be an almost periodic function. If (1.1) has a bounded mild solution on $\mathbb{R}^{+}$, then it has an almost periodic mild solution.

Proof Let $x(\cdot)$ be the mild solution of (1.1) given by (3.2). Since $z(t)$ satisfies (3.1) and $\sigma_{p}(G) \subseteq\{\lambda \in \mathbb{C}: \operatorname{Re}(\lambda) \geq 0\}$, then $z(\cdot)$ is bounded on $\mathbb{R}$. It follows from [38, Theorem 4.2] that $z(\cdot)$ is an almost periodic function. On the other hand, if we denote

$$
Y(t)=\lim _{n \rightarrow \infty} \int_{-\infty}^{t} V^{Q}(t-s) \Pi^{Q}\left(\Gamma^{n} f(s)\right) d s, \quad t \in \mathbb{R}
$$

and

$$
Y^{n}(t)=\int_{-\infty}^{t} V^{Q}(t-s) \Pi^{Q}\left(\Gamma^{n} f(s)\right) d s, \quad t \in \mathbb{R}
$$

then

$$
\begin{aligned}
Y^{n}(t+\tau)-Y^{n}(t)= & \int_{-\infty}^{t+\tau} V^{Q}(t+\tau-s) \Pi^{Q}\left(\Gamma^{n} f(s)\right) d s \\
& -\int_{-\infty}^{t} V^{Q}(t-s) \Pi^{Q}\left(\Gamma^{n} f(s)\right) d s \\
= & \int_{-\infty}^{t} V^{Q}(t-s) \Pi^{Q}\left(\Gamma^{n}(f(s+\tau)-f(s))\right) d s .
\end{aligned}
$$

Let $\varepsilon>0$ and let $P_{\varepsilon}$ be a relatively dense set in $\mathbb{R}$ for which the estimate (2.13) is fulfilled. Using that $V^{Q}(t)$ is a uniformly stable semigroup, there exist constants $M_{1} \geq 1$ and $\alpha>0$ such that $\left\|V^{Q}(t)\right\| \leq M_{1} e^{-\alpha t}$ for $t \geq 0$. Since $\left\|\Gamma^{n}\right\| \leq 1$, from the preceding expression it follows that

$$
\left\|Y^{n}(t+\tau)-Y^{n}(t)\right\| \leq \frac{M_{2}}{\alpha} \varepsilon,
$$

for some constant $M_{2} \geq 0$. Since this estimate is independent of $n$, we also obtain

$$
\|Y(t+\tau)-Y(t)\| \leq \frac{M_{2}}{\alpha} \varepsilon
$$

which shows that the function $Y(\cdot)$ is almost periodic. We complete the proof by combining these assertions with the expression (3.2).

The condition that the semigroup $T$ is uniformly stable is somewhat demanding. However, we can apply the well established mathematical control theory to relax this condition. Specifically, it is well known that there are many important non-delayed distributed control systems modeled by the equation

$$
x^{\prime}(t)=A x(t)+B u(t)
$$


where $B: \mathbb{C}^{m} \rightarrow X$ is a linear map, which are stabilizable. We refer to [40-42] for a discussion about this subject. These results motivate the following concept.

Definition 3.1 The semigroup $T$ is said to be stabilizable if there exists a compact linear operator $K: X \rightarrow X$ such that the semigroup generated by $A+K$ is uniformly stable.

Corollary 3.1 Assume that semigroup $T(\cdot)$ is stabilizable and the operator $T(t) L$ is compact for $t>0$. Let $f: \mathbb{R} \rightarrow X$ be an almost periodic function. If (1.1) has a bounded mild solution on $\mathbb{R}^{+}$, then it has an almost periodic mild solution.

Proof It follows from our hypotheses that there exists a compact linear operator $K: X \rightarrow$ $X$ such that the semigroup $\widetilde{T}(t)$ generated by $\widetilde{A}=A+K$ is uniformly stable. Equation (1.1) can be written

$$
\begin{aligned}
x^{\prime}(t) & =(A+K) x(t)+\widetilde{L}\left(x_{t}\right)+f(t) \\
& =\widetilde{A} x(t)+\widetilde{L}\left(x_{t}\right)+f(t),
\end{aligned}
$$

where the operator $\widetilde{L}: C \rightarrow X$ is given by

$$
\widetilde{L}(\varphi)=L(\varphi)-K \varphi(0)
$$

Moreover,

$$
\widetilde{T}(t) \widetilde{L}(\varphi)=\widetilde{T}(t) L(\varphi)-\widetilde{T}(t) K \varphi(0) .
$$

Since $K$ is a compact operator, we can assert that the operator defined by the second term on the right hand side of (3.5) defines a compact operator. Similarly, it is well known [32] that

$$
\widetilde{T}(t) x=T(t) x+\int_{0}^{t} \widetilde{T}(t-s) K T(s) x d s
$$

and, arguing as in the proof of Theorem 2.1, we see that $\widetilde{T}(t) L$ is a compact operator. Consequently, $\widetilde{T}(t) \widetilde{L}$ is a compact operator, and we can affirm that (3.4) satisfies the hypotheses of Theorem 3.1.

Combining this result with the stabilizability criteria established in [40-42], we can present some results for the existence of almost periodic solutions of equation (1.1) in terms of the controllability of the system (3.3). The system (3.3) is said to be approximately controllable in finite time if for every $x_{1} \in X$ and $\varepsilon>0$ there exist $t_{1}>0$ and a control function $u \in L^{1}\left(\left[0, t_{1}\right], \mathbb{C}^{m}\right)$ such that $\left\|x\left(t_{1}\right)-x_{1}\right\| \leq \varepsilon$, where $x(\cdot)$ is the mild solution of (3.3) with initial condition $x(0)=0$. In $[40,43]$ the reader can find criteria for the approximate controllability of special classes of systems of type (3.3).

In the next result we assume that there is a topological decomposition $X=X_{0} \oplus X_{1}$, where $X_{i}$ are invariant subspaces under $A$, and $X_{1}$ is a finite dimensional space. Let $T_{i}(t)$ be the restriction of the semigroup $T(t)$ on $X_{i}$ for $i=0,1$. Combining Corollary 3.1 with [44, Corollary 3.33] we obtain the following result. 
Corollary 3.2 Let $f: \mathbb{R} \rightarrow X$ be an almost periodic function. Assume that the following conditions hold:

(a) The semigroup $T_{0}(t)$ is uniformly stable.

(b) The system (3.3) is approximately controllable in finite time.

(c) The operator $T(t) L$ is compact for $t>0$.

(d) Equation (1.1) has a bounded mild solution on $\mathbb{R}^{+}$.

Then (1.1) has an almost periodic mild solution.

In [40-42] the reader can find many systems that satisfy the conditions considered in the statement of Corollary 3.2. Similarly, combining Corollary 3.1 with the results in [45], we get the following consequence of the controllability.

Corollary 3.3 Assume that $X$ is a Hilbert space and $T(t)$ is a contraction semigroup such that $T\left(t_{0}\right)$ is compact for some $t_{0}>0$. Let $f: \mathbb{R} \rightarrow X$ be an almost periodic function. Assume further that the following conditions are fulfilled:

(a) The system (3.3) is approximately controllable in finite time.

(c) The operator $T(t) L$ is compact for $t>0$.

(d) Equation (1.1) has a bounded mild solution on $\mathbb{R}^{+}$.

Then (1.1) has an almost periodic mild solution.

Proof It follows from [45, Theorem 3.4.1] that the semigroup $S(t)$ generated by $A-B B^{*}$ is strongly stable. Since

$$
S(t) x=T(t) x-\int_{0}^{t} S(t-s) B B^{*} T(s) x d s,
$$

we see that $S\left(t_{0}\right)$ is compact. Using now [45, Theorem 1.4.6] we find that $S(t)$ is uniformly stable. Therefore, the semigroup $T(t)$ is stabilizable, and the assertion is a consequence of Corollary 3.1 .

\section{Applications}

We initially consider systems governed by a partial differential equation of first order. This type of equations describe interesting phenomena such as transport models [46] or population models with age distribution [47]. Here we consider a simplified system with delay described by the equation

$$
\begin{aligned}
& \frac{\partial w(t, \xi)}{\partial t}+\frac{\partial w(t, \xi)}{\partial \xi}+\alpha w(t, \xi)+\int_{-\infty}^{\infty} g(\xi, \eta) w(t-r, \eta) d \eta=\tilde{f}(t, \xi) \\
& w(\theta, \xi)=\varphi(\theta, \xi)
\end{aligned}
$$

for $\xi \in \mathbb{R}, t \geq 0$, and $-r \leq \theta \leq 0$, where $\alpha, r>0$ and $g, \tilde{f}, \varphi$ are functions that satisfy appropriate conditions that will be specified later. It is well known [48, Example 4.6.1] that the initial value problem

$$
\begin{aligned}
& \frac{\partial w(t, \xi)}{\partial t}+\frac{\partial w(t, \xi)}{\partial \xi}+\alpha w(t, \xi)=0, \quad \xi \in \mathbb{R}, t \geq 0 \\
& w(0, \xi)=h(\xi), \quad \xi \in \mathbb{R}
\end{aligned}
$$


can be modeled as an abstract Cauchy problem in the space $X=L^{2}(\mathbb{R})$. For this reason, in what follows we will assume that $h \in X, f: \mathbb{R} \rightarrow X$ given by $f(t)=\tilde{f}(t, \cdot)$ is a bounded continuous function, and that $\varphi \in C([-r, 0], X)$, where as usual we have identified $\varphi(\theta)(\xi)=$ $\varphi(\theta, \xi)$. Let $A$ be the operator

$$
A z(\xi)=-\frac{d z(\xi)}{d \xi}-\alpha z(\xi)
$$

on the domain $D(A)=H^{1}(\mathbb{R})$. The operator $A$ is the infinitesimal generator of a strongly continuous group $T(t)$ on $X$ given by

$$
T(t) z(\xi)=e^{-\alpha t} z(\xi-t), \quad t, \xi \in \mathbb{R} .
$$

Consequently, using the notation $x(t)=w(t, \cdot)$, the problem (4.3)-(4.4) is reduced to the abstract Cauchy problem

$$
\begin{aligned}
& x^{\prime}(t)=A x(t), \\
& x(0)=h .
\end{aligned}
$$

Furthermore, the semigroup $T(t), t \geq 0$, is uniformly stable, and the operator $T(t)$ is not compact because $T(t)$ has bounded inverse $T(-t)$.

We will assume further that $g: \mathbb{R}^{2} \rightarrow \mathbb{R}$ is continuous and

$$
\int_{-\infty}^{\infty} \int_{-\infty}^{\infty}|g(\xi, \eta)|^{2} d \eta d \xi<\infty
$$

Lemma 4.1 Under the above conditions, the linear operator $N: X \rightarrow X$ given by

$$
N z(\xi)=\int_{-\infty}^{\infty} g(\xi, \eta) z(\eta) d \eta
$$

is compact.

Proof For each $n \in \mathbb{N}$, we define $N_{n}: X \rightarrow X$ by

$$
N_{n} z(\xi)=\int_{-n}^{n} g(\xi, \eta) z(\eta) d \eta, \quad-n \leq \xi \leq n,
$$

and $N_{n} z(\xi)=0$ for $|\xi|>n$. It follows from [49, Proposition 9.5.2] that $N_{n}$ is a compact operator. Next we prove that $N_{n} \rightarrow N$ as $n \rightarrow \infty$ for the norm of operators. In fact, for $z \in X$ we have

$$
\begin{aligned}
\left\|N z-N_{n} z\right\|^{2}= & \int_{-\infty}^{\infty}\left|N z(\xi)-N_{n} z(\xi)\right|^{2} d \xi \\
= & \int_{-\infty}^{-n}\left|\int_{-\infty}^{\infty} g(\xi, \eta) z(\eta) d \eta\right|^{2} d \xi+\int_{-n}^{n} \mid \int_{-\infty}^{-n} g(\xi, \eta) z(\eta) d \eta \\
& +\left.\int_{n}^{\infty} g(\xi, \eta) z(\eta) d \eta\right|^{2} d \xi+\int_{n}^{\infty}\left|\int_{-\infty}^{\infty} g(\xi, \eta) z(\eta) d \eta\right|^{2} d \xi
\end{aligned}
$$




$$
\begin{aligned}
\leq & \int_{-\infty}^{-n} \int_{-\infty}^{\infty}|g(\xi, \eta)|^{2} d \eta d \xi\|z\|^{2}+2 \int_{-n}^{n} \int_{-\infty}^{-n}|g(\xi, \eta)|^{2} d \eta d \xi\|z\|^{2} \\
& +2 \int_{-n}^{n} \int_{n}^{\infty}|g(\xi, \eta)|^{2} d \eta d \xi\|z\|^{2}+\int_{n}^{\infty} \int_{-\infty}^{\infty}|g(\xi, \eta)|^{2} d \eta d \xi\|z\|^{2} .
\end{aligned}
$$

Let $D_{n}=\{(\xi, \eta): \xi, \eta \in[-n, n]\}$. The above estimate shows that

$$
\left\|N-N_{n}\right\|^{2} \leq 2 \iint_{\mathbb{R}^{2} \backslash D_{n}}|g(\xi, \eta)|^{2} d \eta d \xi \rightarrow 0, \quad n \rightarrow \infty,
$$

by Lebesgue's dominated convergence theorem. This implies the assertion.

This result also holds for some functions $g$ discontinuous (the interested reader can consult [50, Proposition V.4.1]).

We define $L: C([-r, 0], X) \rightarrow X$ by $L(\psi)=-N \psi(-r)$. By Lemma 4.1 we see that $L$ is a compact linear map. With this construction, the original system (4.1)-(4.2) is represented by the abstract system

$$
\begin{aligned}
& x^{\prime}(t)=A x(t)+L\left(x_{t}\right)+f(t), \\
& x_{0}=\varphi,
\end{aligned}
$$

and as a consequence of Theorem 3.1 we get the following property.

Corollary 4.1 Under the preceding conditions, iff is almost periodic and (4.1)-(4.2) has a bounded mild solution on $\mathbb{R}^{+}$, then it has an almost periodic solution.

As a second application, we apply our results to study the existence of almost periodic solutions of the wave equation with delay. To establish a general result, we consider an abstract version of the wave equation.

Let $H$ be a real Hilbert space. Following [44, Example 2.16] we consider the abstract wave equation

$$
\begin{aligned}
& x^{\prime \prime}(t)+\beta x^{\prime}(t)+A x(t)=0, \\
& x(0)=x^{0}, \quad x^{\prime}(0)=x^{1},
\end{aligned}
$$

where $x(t) \in H, \beta>0$ and $A$ is a positive self adjoint operator with domain $D(A)$ such that

$$
\langle A x, x\rangle \geq k\|x\|^{2}, \quad \forall x \in D(A)
$$

for some constant $k>0$.

Introducing the Hilbert space $\mathcal{H}=D\left(A^{1 / 2}\right) \times H$ with inner product

$$
\left\langle\left[\begin{array}{l}
x^{1} \\
y^{1}
\end{array}\right],\left[\begin{array}{l}
x^{2} \\
y^{2}
\end{array}\right]\right\rangle=\left\langle A^{1 / 2} x^{1}, A^{1 / 2} x^{2}\right\rangle+\left\langle y^{1}, y^{2}\right\rangle,
$$

we can write (4.5) as the first order system

$$
w^{\prime}(t)=\mathcal{A} w(t)
$$


where $w(t)=\left[\begin{array}{c}x(t) \\ x^{\prime}(t)\end{array}\right] \in \mathcal{H}$ and $\mathcal{A}=\left[\begin{array}{cc}0 & I \\ -A & -\beta\end{array}\right]$ is defined on $D(\mathcal{A})=D(A) \times D\left(A^{1 / 2}\right)$. Then $\mathcal{A}$ generates a strongly continuous group $G(t)$ on $\mathcal{H}$. Consequently, $G(t)$ is not compact.

We assume also that $\mu \in \rho(-A)$ and $\|R(\mu,-A)\| \leq \frac{C}{|\mu|}$ for $\operatorname{Re}(\mu)>0$. Hence, for every $\lambda \in \mathbb{C}$ with $\operatorname{Re}(\lambda)>0$ we have $\lambda \in \rho(\mathcal{A})$,

$$
(\lambda I-\mathcal{A})^{-1}=\left[\begin{array}{cc}
(\lambda+\beta) R(\lambda(\lambda+\beta),-A) & R(\lambda(\lambda+\beta),-A) \\
-A R(\lambda(\lambda+\beta),-A) & \lambda R(\lambda(\lambda+\beta),-A)
\end{array}\right],
$$

and $\left\|(\lambda I-\mathcal{A})^{-1}\right\| \leq C$, where $C>0$ is a generic constant. Thus, under the above conditions, it follows from [31, Theorem V.1.11] that $G(t)$ is uniformly stable.

We consider now the inhomogeneous wave equation

$$
x^{\prime \prime}(t)+\beta x^{\prime}(t)+A x(t)=f(t) .
$$

Using the previous transformation, we can reduce (4.7) to the first order equation

$$
w^{\prime}(t)=\mathcal{A} w(t)+\tilde{f}(t)
$$

where $\tilde{f}(t)=\left[\begin{array}{c}0 \\ f(t)\end{array}\right]$.

Finally we consider the wave equation with delay

$$
x^{\prime \prime}(t)+\beta x^{\prime}(t)+A x(t)=f(t)+L_{1}\left(x_{t}\right)
$$

where $L_{1}: C([-r, 0], H) \rightarrow H$ is a bounded linear operator. Using the previous transformation, we can reduce (4.8) to the first order equation with delay

$$
w^{\prime}(t)=\mathcal{A} w(t)+L\left(w_{t}\right)+\tilde{f}(t)
$$

where $L: C([-r, 0], \mathcal{H}) \rightarrow \mathcal{H}$ is given by

$$
L\left(\left[\begin{array}{l}
\varphi \\
\psi
\end{array}\right]\right)=\left[\begin{array}{c}
0 \\
L_{1}(\varphi)
\end{array}\right] .
$$

The following property is a direct consequence of Theorem 3.1.

Corollary 4.2 Under the above conditions, let $f: \mathbb{R} \rightarrow H$ be an almost periodic function. Assume that $L_{1}$ is a compact operator, and that (4.8) has a bounded mild solution on $\mathbb{R}^{+}$. Then (4.8) has an almost periodic mild solution.

Proof It is clear that $L$ is a compact operator so that $G(t) L$ is also compact for all $t>0$.

To complete this application, next we will present a pair of concrete examples of compact linear operators $L_{1}: C([-r, 0], H) \rightarrow H$.

(i) Let $K: H \rightarrow H$ be a compact linear operator. We fix $\theta_{0} \in[-r, 0]$ and define $L_{1}(\varphi)=$ $K \varphi\left(\theta_{0}\right)$ for $\varphi \in C([-r, 0], H)$. It is immediate that $L_{1}$ is a compact linear operator. 
(ii) Let $H$ be a separable Hilbert space with orthonormal basis $\left\{z_{n}: n \in \mathbb{N}\right\}$. Let $\eta_{n}$ : $[-r, 0] \rightarrow \mathbb{C}$ for $n \in \mathbb{N}$ be a function with bounded variation $V\left[\eta_{n}\right]$. We assume that $\sum_{n=1}^{\infty} V\left[\eta_{n}\right]^{2}<\infty$. Let $L_{1}$ given by

$$
L_{1}(\varphi)=\sum_{n=1}^{\infty} \int_{-r}^{0} d_{\theta} \eta_{n}(\theta)\left\langle\varphi(\theta), z_{n}\right| z_{n}, \quad \varphi \in C([-r, 0], H) .
$$

It is not difficult to verify that $L_{1}$ is a compact linear operator.

Finally, we present an application in which the original semigroup is not uniformly stable. A large number of concrete systems can be formulated in the following abstract form. Let $H$ be a separable Hilbert space with orthonormal basis $\left\{z_{n}: n \in \mathbb{N}\right\}$. Let $\left(\lambda_{n}\right)_{n}$ be a sequence of complex numbers such that $\sup _{n \in \mathbb{N}} \operatorname{Re}\left(\lambda_{n}\right)<\infty$. We consider the operator $A: D(A) \subseteq H \rightarrow H$ given by

$$
A z=\sum_{n=1}^{\infty} \lambda_{n}\left\langle z, z_{n}\right\rangle z_{n}, \quad z \in D(A)
$$

where $D(A)=\left\{z \in H: \sum_{n=1}^{\infty}\left|\lambda_{n}\right|^{2}\left|\left\langle z, z_{n}\right\rangle\right|^{2}<\infty\right\}$. It is well known that $A$ generates a strongly continuous semigroup $T(t)$ given by

$$
T(t) z=\sum_{n=1}^{\infty} e^{\lambda_{n} t}\left\langle z, z_{n}\right\rangle z_{n}, \quad z \in H
$$

which generally is not compact and is not uniformly stable. Let $\beta>0$. We decompose $\mathbb{N}=I \cup J$, where $I=\left\{n \in \mathbb{N}: \operatorname{Re}\left(\lambda_{n}\right)>-\beta\right\}$ and $J=\mathbb{N} \backslash I$. We assume that $\lambda_{n} \rightarrow 0$ as $n \rightarrow \infty$, $n \in I$. We define the operator $K: H \rightarrow H$ by $K z=\sum_{n \in I} \lambda_{n}\left\langle z, z_{n}\right\rangle z_{n}$. It is easy to verify that $K$ is a compact linear operator. Moreover, the semigroup $S(t)$ generated by $A-K$ is given by

$$
S(t) z=\sum_{n \in J} e^{\lambda_{n} t}\left\langle z, z_{n}\right\rangle z_{n}, \quad z \in H,
$$

and $\|S(t) z\| \leq e^{-\beta t}\|z\|$ for $t \geq 0$, which shows that $S(t)$ is uniformly stable. Hence, we can apply Corollary 3.1 to conclude that under the preceding conditions if the operator $T(t) L$ is compact for $t>0, f: \mathbb{R} \rightarrow H$ is an almost periodic function, and (1.1) has a bounded mild solution on $\mathbb{R}^{+}$, then it has an almost periodic mild solution.

\section{Conclusion}

In this work we have studied the existence of almost periodic solutions of ARFDEs where the operator acting on the delay term is bounded. Somewhat superficially, our results are able to reduce the existence of almost periodic solutions of the inhomogeneous equation to the possibility to control or stabilize the system without delay.

The authors declare that they have no competing interests. 


\section{Author details}

'Departmento de Matemática, Universidad de Santiago-USACH, Casilla 307, Correo-2, Santiago, Chile. ${ }^{2}$ Departmento of Matemática, Universidade Federal of Pernambuco, Av. Prof. Luiz Freire, S/N, Recife, PE 50540-740, Brazil. ${ }^{3}$ Departamento de Matematica, Centro de Ciências Exatas e Tecnologia, Universidade Federal de Sergipe, Av Vereador Olimpo Grande s/n Itabaiana, SE 49500000, Brazil.

\section{Acknowledgements}

The authors acknowledge the disposition of the reviewer to review the work as well as his numerous comments and suggestions that greatly improved the original text. The first author was supported partially by CONICYT under Grant FONDECYT 1130144 and DICYT-USACH. The second author was supported partially by CNPq/Brazil under Grant 478053/2013-4.

Received: 15 October 2014 Accepted: 27 January 2015 Published online: 12 February 2015

\section{References}

1. Hino, Y, Murakami, S: A generalization of processes and stabilities in abstract functional differential equations. Funkc. Ekvacioj 41, 235-255 (1998)

2. Murakami, S: Periodic solutions of some functional differential equations with diffusion. Funkc. Ekvacioj 40, 1-17 (1997)

3. Murakami, S, Nagabuchi, Y: Invariant manifolds for abstract functional differential equations and related Volterra difference equations in a Banach space. Funkc. Ekvacioj 50, 133-170 (2007)

4. Wu, J: Theory and Applications of Partial Functional Differential Equations. Springer, New York (1996)

5. Higham, DJ, Sardar, TK: Existence and stability of fixed points for a discretised nonlinear reaction-diffusion equation with delay. Appl. Numer. Math. 18, 155-173 (1995)

6. Gu, W, Wang, P: A Crank-Nicolson difference scheme for solving a type of variable coefficient delay partial differential equations. J. Appl. Math. 2014, Article ID 560567 (2014)

7. Jin, YF, Jiang, J, Hou, CM, Guan, DH: New difference scheme for general delay parabolic equations. J. Inf. Comput. Sci. 9(18), 5579-5586 (2012)

8. Sardar, TK, Higham, DJ: Dynamics of constant and variable stepsize methods for a nonlinear population model with delay. Appl. Numer. Math. 24, 425-438 (1997)

9. Tanabe, H: Functional Analytic Methods for Partial Differential Equations. Dekker, New York (1997)

10. Ashyralyev, A, Sobolevskii, PE: On the stability of the delay differential and difference equations. Abstr. Appl. Anal. 6(5), 267-297 (2001)

11. Ashyralyev, A, Sobolevskii, PE: New Difference Schemes for Partial Differential Equations. Birkhäuser, Basel (2014)

12. Di Blasio, G: Delay differential equations with unbounded operators acting on delay terms. Nonlinear Anal. TMA 52(1), 1-18 (2003)

13. Shakhmurov, VB, Sahmurova, A: Abstract parabolic problems with parameter and application. Appl. Math. Comput. 219(17), 9561-9571 (2013)

14. Ashyralyev, A, Agirseven, D: Finite difference method for delay parabolic equations. In: Numerical Analysis and Applied Mathematics ICNAAM 2011: International Conference on Numerical Analysis and Applied Mathematics. AIP Conference Proceedings, vol. 1389, pp. 573-576 (2011)

15. Agirseven, D: Approximate solutions of delay parabolic equations with the Dirichlet condition. Abstr. Appl. Anal. 2012, Article ID 682752 (2012)

16. Ashyralyev, A, Agirseven, D: On convergence of difference schemes for delay parabolic equations. Comput. Math Appl. 66(7), 1232-1244 (2013)

17. Ashyralyev, A, Agirseven, D: Well-posedness of delay parabolic difference equations. Adv. Differ. Equ. 2014, Article ID 18 (2014)

18. Ashyralyev, A, Agirseven, D: Well-posedness of delay parabolic equations with unbounded operators acting on delay terms. Bound. Value Probl. 2014, Article ID 126 (2014)

19. Ashyralyev, A, Agirseven, D: Note on the stability of delay parabolic equations with unbounded operators acting on delay terms. Electron. J. Differ. Equ. 2014, 160 (2014)

20. Ashyralyev, A, Agirseven, D: Stability of delay parabolic difference equations. Filomat 28(5), 995-1006 (2014)

21. Hino, Y, Naito, T, Van Minh, N, Shin, JS: Almost Periodic Solutions of Differential Equations in Banach Spaces. Taylor \& Francis, London (2002)

22. Boukli-Hacene, N, Ezzinbi, K: Weighted pseudo almost periodic solutions for some partial functional differential equations. Nonlinear Anal. 71, 3612-3621 (2009)

23. Cuevas, C, Hernández, EM: Pseudo-almost periodic solutions for abstract partial functional differential equations. Appl. Math. Lett. 22, 534-538 (2009)

24. Ezzinbi, K, Fatajou, S, N'Guérékata, GM: Pseudo almost automorphic solutions for some partial functional differential equations with infinite delay. Appl. Anal. 87, 591-605 (2008)

25. Ezzinbi, K, N'Guérékata, GM: Almost automorphic solutions for some partial functional differential equations. J. Math. Anal. Appl. 328, 344-358 (2007)

26. Henríquez, HR: Asymptotically almost periodic solutions of abstract retarded functional differential equations of first order. Nonlinear Anal., Real World Appl. 10, 2441-2454 (2009)

27. Liu, Q, Van Minh, N, N'Guérékata, G, Yuan, R: Massera type theorems for abstract functional differential equations. Funkc. Ekvacioj 51, 329-350 (2008)

28. Liu, Q, Yuan, R: Asymptotic behavior of solutions to abstract functional differential equations. J. Math. Anal. Appl. 356, 405-417 (2009)

29. Ruess, WM: Compactness and asymptotic stability for solutions of functional differential equations with infinite delay. In: Ferreyra, G, Ruiz Goldstein, G, Neubrander, F (eds.) Evolution Equations. Lect. Notes Pure and Appl. Math., vol. 168, pp. 361-374. Dekker, New York (1995)

30. Zhang, $L, X u, Y$ : Weighted pseudo almost periodic solutions for functional differential equations. Electron. J. Differ. Equ. 2007, 146 (2007) 
31. Engel, KJ, Nagel, R: One-Parameter Semigroups for Linear Evolution Equations. Springer, New York (2000)

32. Pazy, A: Semigroups of Linear Operators and Applications to Partial Differential Equations. Springer, New York (1983)

33. Goldberg, S: Unbounded Linear Operator. Dover, New York (1985)

34. Travis, CC, Webb, GF: Existence and stability for partial functional differential equations. Trans. Am. Math. Soc. 200 395-418 (1974)

35. Henríquez, HR: On non-exact controllable systems. Int. J. Control 42, 71-83 (1985)

36. Hino, Y, Murakami, S, Naito, T, Van Minh, N: A variation of constants formula for abstract functional differential equations in the phase space. J. Differ. Equ. 179, 336-355 (2002)

37. Hale, J, Verduyn Lunel, SM: Introduction to Functional Differential Equations. Springer, New York (1993)

38. Corduneanu, C: Almost Periodic Functions. Chelsea, New York (1989)

39. Zaidman, SD: Almost-Periodic Functions in Abstract Spaces. Pitman, London (1985)

40. Curtain, RF, Zwart, HJ: An Introduction to Infinite-Dimensional Linear Systems Theory. Springer, New York (1995)

41. Pritchard, AJ, Zabczyk, J: Stability and stabilizability of infinite dimensional systems. SIAM Rev. 23, 25-52 (1981)

42. Triggiani, R: On the stabilizability problem in Banach space. J. Math. Anal. Appl. 52, 383-403 (1975)

43. Henríquez, HR: Approximate controllability of linear distributed control systems. Appl. Math. Lett. 21, 1041-1045 (2008)

44. Curtain, RF, Pritchard, AJ: Infinite Dimensional Linear Systems Theory. Lect. Notes in Control and Information Sciences, vol. 8. Springer, Berlin (1978)

45. Benchimol, CD: Feedback stabilizability in Hilbert spaces. Appl. Math. Optim. 4, 225-248 (1978)

46. Salsa, S: Partial Differential Equations in Action. From Modelling to Theory. Springer, Milan (2008)

47. Murray, JD: Mathematical Biology. I. An Introduction, 3rd edn. Springer, Berlin (2002)

48. Balakrishnan, AV: Applied Functional Analysis, 2nd edn. Springer, New York (1981)

49. Edwards, RE: Functional Analysis. Theory and Applications. Dover, New York (1995)

50. Martin, RH: Nonlinear Operators and Differential Equations in Banach Spaces. Krieger, Melbourne (1987)

\section{Submit your manuscript to a SpringerOpen ${ }^{\circ}$ journal and benefit from:}

- Convenient online submission

Rigorous peer review

- Immediate publication on acceptance

Open access: articles freely available online

- High visibility within the field

- Retaining the copyright to your article 